\title{
Cost-Effectiveness Analysis of Human Papillomavirus Vaccination in Adolescent Girls in Taiwan
}

\author{
Chao-Hsiun Tang1, Wen Fang Cheng ${ }^{2,3,4}$, Jhih-Hua Jiang1, San Lin You, \\ Lee-Wen Huang ${ }^{6}$, Jui-Yu Hsieh ${ }^{7}$, Piyali Mukherjee ${ }^{8}$, Georges Van Kriekinge, \\ Christa Lee ${ }^{8 *}$
}

\begin{abstract}
Objective: Three vaccines are available to Taiwanese young girls for cervical cancer (CC) prevention. Here we evaluate the cost-effectiveness of the two-dose (2D) AS04-adjuvanted HPV-16/18 vaccine (2D-AS04-HPV$16 / 18 v$ ) + screening compared with a screening programme alone, with 2D human papillomavirus 6/11/16/18 vaccine (2D-4vHPVv)+screening, and with 2D/three-dose (3D) human papillomavirus 6/11/16/18/31/33/45/52/58 vaccine $(9 \mathrm{vHPVv})+$ screening, for Taiwan universal mass vaccination. Methods: A static Markov cohort model simulated the natural history of human papillomavirus (HPV) infection and CC screening for a 12-year-old cohort of Taiwanese girls $(\mathrm{N}=120,000)$. The model ran in 1-year cycles over the cohort's lifetime. Vaccine efficacy irrespective of HPV type was considered in the analysis for each vaccine. Input data were obtained from published literature, local databases, government reports and websites, and expert opinion. The analysis incorporated direct medical costs only, with an annual discount rate of $3.0 \%$. The threshold was determined as 1 Gross Domestic Product per capita (New Taiwan dollar [NT\$] 727,818; year 2016). Results: The 2D-AS04-HPV-16/18v+screening yielded 0.0365 quality-adjusted life year (QALY) gained at an additional cost of NT\$ 5,770 per person compared with the screening programme alone. This resulted in an incremental cost-effectiveness ratio well below the threshold. Compared with $2 \mathrm{D}-4 \mathrm{vHPVv}+$ screening and 2D/3D-9vHPVv+screening, discounted results demonstrated additional QALYs gained at lower cost for 2D-AS04-HPV$16 / 18 \mathrm{v}+$ screening, making it dominant over both $2 \mathrm{D}-4 \mathrm{vHPVv}+$ screening and $2 \mathrm{D} / 3 \mathrm{D}-9 \mathrm{vHPV}+$ screening. Conclusions: Vaccinating Taiwanese girls with $2 \mathrm{D}-\mathrm{AS} 04-\mathrm{HPV}-16 / 18 \mathrm{v}$ in addition to screening to prevent CC is cost-effective compared with using a screening programme alone and the dominant option compared with $2 \mathrm{D}-4 \mathrm{vHPVv}+\mathrm{screening}$ and 2D/3D-9vHPVv+screening.
\end{abstract}

Keywords: HPV vaccination- cervical cancer- cost- effectiveness- Taiwan

Asian Pac J Cancer Prev, 20 (5), 1377-1387

\section{Introduction}

Cervical cancer (CC) had a high incidence in Taiwan, accounting for more than $30 \%$ of all cancer cases and being the third most common cause of cancer death among Taiwanese women in the 1990s (Wang and Lin, 1996). In 1995, a nationwide annual Papanicolaou (Pap) screening programme was put in place, allowing the detection of cervical abnormalities at an earlier stage and the timely provision of treatment. Thanks to this programme, incidence and mortality rates decreased by $46 \%$ and $47 \%$ in 2006 and 2007, respectively (Lee et al., 2012). However, compliance with cervical screening has remained sub-optimal in Taiwan, with the annual screening coverage in 2007 and 2014 reported at $27.4 \%$ and $27.6 \%$ respectively (Bureau of Health Promotion, 2007; Health Promotion Administration - Ministry of Health and Welfare - Taiwan, 2014).

Human papillomavirus (HPV) is the necessary cause of CC, detected in $>95 \%$ of CC cases (Muñoz et al., 2003; Walboomers et al., 1999). Persistent infections with oncogenic HPV strains (most frequently HPV-16 and HPV18) may lead to the development of pre-cancerous cervical intraepithelial neoplasia $(\mathrm{CIN})$ grade 1 which over time may progress to grade $2 \mathrm{CIN}$ (CIN2), grade $3 \mathrm{CIN}$ (CIN3) and CC (Castellsague, 2008; Demarteau et al., 2012; Köse and Naki, 2014). On the other hand, non-oncogenic HPV types have been associated with low-grade cervical

${ }^{1}$ School of Health Care Administration, Taipei Medical University, ${ }^{2}$ Department of Obstetrics and Gynecology, ${ }^{3}$ Graduate Institute of Clinical Medicine, ${ }^{4}$ Graduate Institute of Oncology, College of Medicine, National Taiwan University, ${ }^{6}$ Department of Obstetrics and Gynecology, Shin Kong Wu Ho-Su Memorial Hospital, ${ }^{7}$ GSK, Taipei, ${ }^{5}$ School of Medicine, College of Medicine, Big Data Research Centre, Fu-Jen Catholic University, New Taipei City, Taiwan, ${ }^{8}$ GSK, Singapore, Singapore, ${ }^{9}$ GSK, Wavre, Belgium. *For Correspondence: christa.x.lee@gsk.com. Chao-Hsiun Tang and Wen Fang Cheng have equal contribution in this study. 
lesions and genital warts (GW) (Demarteau et al., 2012). Given the sub-optimal CC screening coverage in Taiwan and HPV infection being the necessary cause of CC, the risk of $\mathrm{CC}$ can be substantially reduced through a combination of HPV vaccination and CC screening programmes (Demarteau et al., 2012; Paavonen et al., 2009). The World Health Organization (WHO) recognises the importance of $\mathrm{CC}$ as a global public health problem and recommends HPV vaccines are included in national immunisation programmes (World Health Organization, 2017). Vaccination programmes have an important impact at the population level, as there is evidence of a consequent reduction in high-grade cervical abnormalities and in prevalence of oncogenic HPV types among young women (Cameron et al., 2017; Gertig et al., 2013; Kavanagh et al., 2017; Powell et al., 2012; Tabrizi et al., 2014; World Health Organization, 2017).

There are three HPV vaccines currently available in Taiwan, protecting Taiwanese young girls against CC: a two-dose (2D) AS04-adjuvanted HPV-16/18 vaccine (AS04-HPV-16/18v, Cervarix, GSK, Belgium) containing virus-like particles (VLPs) for oncogenic HPV types 16 and 18, a 2D human papillomavirus 6/11/16/18 vaccine (4vHPVv, Gardasil, Merck, USA) containing two additional VLPs for non-oncogenic HPV types 6 and 11, and a 2D/three-dose (3D) human papillomavirus 6/11/16/18/31/33/45/52/58 vaccine (9vHPVv, Gardasil 9, Merck, USA) containing VLPs for five additional oncogenic HPV types.

Cost-effectiveness of HPV vaccines has been previously explored in the Taiwanese context, comparing vaccination + screening versus screening alone and AS04-HPV-16/18v versus 4vHPVv, in combination with screening (Dasbach et al., 2008; Demarteau et al., 2012; Liu et al., 2010; Rogoza et al., 2008; Suárez et al., 2008). The studies demonstrated that both AS04-HPV-16/18v and $4 \mathrm{vHPVv}$, in combination with screening, are cost-effective compared with screening alone (Dasbach et al., 2008; Liu et al., 2010; Rogoza et al., 2008; Suárez et al., 2008). When the two vaccines were compared, AS04-HPV$16 / 18 \mathrm{v}$ resulted in better health outcomes at lower cost than $4 \mathrm{vHPVv}$, in combination with screening (Demarteau et al., 2012). However, the studies were based on a 3-dose (3D) schedule for both vaccines. Since the introduction of the 2D schedules for AS04-HPV-16/18v and 4vHPV $v$ in 2015 and the newly-approved 9vHPVv with 2D and 3D schedules in Taiwan (2016/2017), no published study has reported the relative cost-effectiveness of these vaccines in the Taiwanese context.

The objective of this study is to assess the costeffectiveness of vaccination with 2D-AS04-HPV-16/18v combined with screening, compared with: (i) screening alone; (ii) 2D-4vHPVv combined with screening; (iii) 2D-9vHPVv combined with screening; (iv) 3D-9vHPVv combined with screening.

\section{Materials and Methods}

Model overview

The analysis used a previously published Markov cohort model reflecting the natural history of oncogenic
HPV infection to invasive CC and of non-oncogenic HPV infection to GW and/or CIN1, where fixed annual transition probabilities determine cohort movement between health states (Demarteau et al., 2012; Suárez et al., 2008).

In the current analysis, two additional comparators were incorporated into the model: $2 \mathrm{D}-9 \mathrm{vHPVv}$ and $3 \mathrm{D}-9 \mathrm{vHPVv}$ combined with screening.

\section{Study population}

Based on demographic data from Taiwan, the model included a cohort $(\mathrm{N}=120,000)$ of girls aged 12 years old (i.e. $7^{\text {th }}$ grade) (Department of Household Registration - M.O.I., 2016). This age group of girls was targeted because they are representative of the female population prior to HPV exposure and thus are expected to benefit most from the vaccination. They are also the targeted group in many parts of the world with existing school-based vaccination programmes.

\section{Cycle time and time horizon}

The model was run for 95 years in 1-year cycles to cover the total lifetime of the cohort (i.e., until 107 years of age). Annual transition probabilities determined how the cohort moves from one health state to the next in every cycle.

\section{Perspective}

The analysis was undertaken from the perspective of the Taiwanese government. Therefore, only direct medical costs were included in the model.

\section{Discounting}

In accordance with the Taiwanese pharmacoeconomic recommendations and local expert opinion, a discount rate of $3.0 \%$ was applied to both costs and health outcomes in the base-case analysis (Center for Drug Evaluation, 2014; International Society for Pharmacoeconomics and Outcome Research, 2006). In univariate sensitivity analysis, a range of $0.0-5.0 \%$ and $0.0-3.0 \%$ was explored for costs and health outcomes, respectively.

\section{Threshold}

Based on the WHO guidelines and local expert opinion, 1 Gross Domestic Product (GDP) per capita was deemed an appropriate threshold to assess cost-effectiveness in Taiwan (Bertram et al., 2016). Therefore, the willingness-to-pay (WTP) threshold was established at 727,818 New Taiwan dollar (NT\$) per quality-adjusted life year (QALY) gained based on the published 2016 GDP per capita in Taiwan (Directorate General of Budget - Accounting and Statistics - Executive Yuan - R.O.C. (Taiwan), 2017).

\section{Data input and assumptions}

Model inputs were derived from local data where possible, and consulted and validated by local experts.

\section{Vaccine effectiveness}

The model used vaccine efficacy estimates irrespective of HPV type as proxies of vaccine effectiveness (Table 
1). The efficacy estimates were based on product label in Taiwan as well as clinical trial data for girls and women who were "naïve" to HPV infection at study entry.

However, data required to estimate $9 \mathrm{vHPVv}$ vaccine effectiveness against $\mathrm{CC}$ (i.e. vaccine efficacy against CIN3 or higher $[\mathrm{CIN} 3+]$ ) were limited. Therefore, this parameter was projected based on available data from Joura et al. (2014) and the clinical trial synopsis of 9vHPVv (Merck Sharp and Dohme Corp., 2015). Joura et al. (2014) estimated an expected vaccine efficacy with $9 \mathrm{vHPV}$ to be $43-55 \%, 70-78 \%$ and $85-91 \%$ against CIN1, CIN2 and CIN3, respectively. To estimate the potentially observable vaccine efficacy against CIN3/CIN3+, the expected vaccine efficacy estimates for CIN1 and CIN2 were compared with their observed efficacy estimates from the clinical trial and an average ratio of $90.5 \%$ between the observed versus expected efficacies was calculated. This average ratio was applied to the average expected efficacy of $88.0 \%$ for 9vHPVv against CIN3 (Merck Sharp and Dohme Corp., 2015), leading to an adjusted vaccine efficacy of $79.6 \%$ for $9 \mathrm{vHPVv}$ against CIN3/CIN3+.

Moreover, the proxy effectiveness against GW was assumed in the base-case to be $0.0 \%$ for AS04-HPV-16/18v according to its label in Taiwan (GSK Taiwan, 2016). For $4 v H P V v$ and $9 v H P V v$, vaccine effectiveness irrespective of the causative HPV type against GW was estimated at $82.8 \%$ and $94.6 \%$, respectively (Giuliano et al., 2014; Munoz et al., 2010). Vaccine effectiveness was assumed to be life-long in the base-case analysis.

Further details about calculation of vaccine effectiveness are provided in Supplementary Material 1.

\section{Transition probabilities}

Transition probabilities used in the model reflect the natural history of the disease. These data were mainly taken from previous Taiwan HPV cost-effectiveness analyses (Demarteau et al., 2012; Suárez et al., 2008) where the following data have been updated with local or more recent available data: progression from non-oncogenic HPV to CIN1 or GW, from persistent CIN2/3 to CC and from CC to death, regression from $\mathrm{CC}$ to no HPV and GW resistance (Health Promotion Administration - Ministry of Health and Welfare - Taiwan; Ministry of Health and Welfare - Taiwan, 2013; Sanders and Taira, 2003; Shen et al., 2016; Van de Velde et al., 2007; Woodhall et al., 2011) (Supplementary Material 2).

\section{Epidemiological data}

The epidemiological data incorporated into the model derived from different published sources: CC incidence and mortality from Cancer Registry Interactive System (Health Promotion Administration - Ministry of Health and Welfare - Taiwan) (Table 2); GW incidence from the National Health Insurance Research Database (NHID) (Table 2); HPV prevalence mainly from the published literature (Chao et al., 2008; Chen et al., 2011; Richardson et al., 2003) (personal communication) (Table S 3 in Supplementary Material 3); all-cause mortality probabilities in the general female population from the Ministry of the Interior - Department of Statistics (Table
S 4 in Supplementary Material 3); and distribution of $\mathrm{CC}$ incidence by stage from the Taiwan Cancer Registry Center (2014) (Table S 5 in Supplementary Material 3). Further details on the epidemiological data are provided in Supplementary Material 3.

\section{Vaccination, treatment and screening practices}

The majority of the model inputs reflecting screening, vaccination and treatment practices is specific to Taiwan and was derived from the literature and government reports/websites, and based on local expert opinion (Supplementary Material 4).

\section{Cost data}

Cost data incorporated in the model were mostly derived from patient registries and database analyses (Table 3 and Supplementary Material 5). Private market prices were used for all vaccines, assuming price parity for AS04-HPV-16/18v and 4vHPVv. Cost estimates were inflated to year 2016 by using the medical care services Consumer Price Index (CPI) in Taiwan (National Statistics - Republic of China (Taiwan), 2018).

\section{QALY loss}

Utility decrements for each health state were derived from the published literature (Marcellusi et al., 2015) and adjusted by taking into account the duration of each identified health state (Annemans et al., 2009). Distribution of CC incidence by stage obtained from the Taiwan Cancer Registry Annual report was applied to estimate the overall utility decrement for the 'cancer cured' health state (Goldie et al., 2004; Taiwan Cancer Registry Center, 2014) (Table 4).

\section{Outcomes}

In the model, each health state was associated with a disutility score and a cost. Both disutility scores and costs were combined with the time spent in each health state to estimate the total accrued cost and number of QALYs over the cohort's lifetime.

The primary outcome reported from the analysis was the incremental cost-effectiveness ratio (ICER) when both incremental cost and incremental QALY were positive. When either was negative, differences in cost and health outcomes were reported separately instead.

Other reported outcome measures include: undiscounted number of CC cases, CC deaths, screening-detected CIN cases and GW cases, undiscounted and discounted costs, and undiscounted and discounted life years (LYs) and QALYs gained. All outcomes were reported per person.

\section{Scenario analysis}

Three scenario analyses were conducted around key assumptions and model inputs included in the base-case analysis: limited duration of immunity, higher GW incidence and vaccine tender prices.

\section{Duration of immunity}

There remains an incomplete understanding on the duration of HPV vaccine protection as the evidence on long-term vaccine effectiveness to date is limited (Aregay

Asian Pacific Journal of Cancer Prevention, Vol 201379 
et al., 2013; David et al., 2009; Ferris et al., 2014; Joura et al., 2015; Naud et al., 2014). The possibility of limited duration of immunity was explored. In the scenario analysis, vaccine immunity started to decline 20 years post-vaccination by $30 \%$ over 5 years and then remained constant through the lifetime of the cohort. This scenario analysis was applied to all vaccines and included no booster dose.

\section{GW incidence}

GW incidence data obtained from the NHID were considered as the most robust data available for the Taiwanese population (Ministry of Health and Welfare - Taiwan, 2013). However, given the concern associated with misdiagnosis and the frequent use of self-paid cream in Taiwan to manage GW cases, a scenario analysis was undertaken to examine the impact of using higher GW incidence data as reported from Korea in the analysis (National Evidence-based Healthcare Collaborating Agency (NECA) Study, 2012).

\section{Vaccination costs}

Vaccine tender prices reflect prices per dose, including administration costs, applicable to the Taiwanese government. To account for differences in vaccine prices quoted to the government and in the private market, the effect of using the tender prices (AS04-HPV-16/18v: NT\$ 1,800; 4vHPVv: NT\$ 1,800 and 9vHPVv: NT\$ $2,500)$ instead of private market prices was explored in the scenario analysis (Government e-Procurement System for Health Promotion Administration, Ministry of Health and Welfare; Government e-Procurement System for Public Health Bureau, Chiayi City).

\section{Sensitivity analyses}

Two types of sensitivity analyses were performed: a univariate sensitivity analysis and a probabilistic sensitivity analysis (PSA) to assess the robustness of the model results. Ranges and distributions used in the sensitivity analyses are presented in Supplementary Material 6.

\section{Univariate sensitivity analysis}

A univariate sensitivity analysis was performed to assess the effect of uncertainty around key parameters in the model on the outcomes. The analysis used relevant ranges for each variable such as the minimum and maximum values of the confidence interval or a variation of $20 \%$ higher or lower than the base-case. For example, the proxy effectiveness against $\mathrm{GW}$ varied between 0.0 and $50.9 \%$ in the sensitivity analysis, based on evidence of decline in GW incidence among young women vaccinated with AS04-HPV-16/18v in England (Canvin et al., 2016; Public Health England, 2012; Szarewski et al., 2013; Toft et al., 2014). For the 9vHPVv vaccination cost, a broad range $(120 \%-200 \%)$ of $4 \mathrm{vHPVv}$ vaccination cost was explored given its recent entry in the Taiwanese market (Supplementary Material 6).

\section{Probabilistic sensitivity analysis}

A PSA was performed to further test parameters' uncertainty and to evaluate the overall robustness of the model. Distributions were assigned to parameters for transition probability, disease burden, vaccine effectiveness, cost and utility as informed in Supplementary Material 6. In total, 1,000 samples were generated from the assigned distribution for each comparison.

\section{Results}

Base-case analysis

The incremental results of the base-case analysis for the 2D-AS04-HPV-16/18v compared with screening alone, and with other vaccines (2D-4vHPVv, 2D-9vHPVv and 3D-9vHPVv; applied in addition to screening) are presented in Table 5. Detailed results of the base-case analysis are provided in Supplementary Material 7.

\section{Cases and deaths}

Combined with screening, 2D-AS04-HPV-16/18v prevented more screening-detected CIN2/3 cases, CC cases and $\mathrm{CC}$ deaths per person compared with screening only as well as in comparison with the other vaccines, 2D $-4 v H P V v$ and 2D/3D-9vHPVv combined with screening. However, 2D-4vHPVv+screening averted more GW cases, and 2D/3D-9vHPVv+screening prevented more GW cases and more screening-detected CIN1 cases per person, compared with 2D-AS04-HPV-16/18v+screening (Table 5).

\section{Costs, QALY and ICER}

Compared with screening alone, 2D-

Table 1. Vaccine Effectiveness in HPV-Naive Population Estimated Based on Vaccine Efficacy Irrespective of HPV Type

\begin{tabular}{lccc}
\hline Endpoint in the model & AS04-HPV-16/18v & 4vHPVv & 9vHPVv \\
\hline CC (proxy for CIN3/CIN3+) & $93.2 \%$ & $43.0 \%$ (Munoz et al., 2010) & $\begin{array}{c}\text { 79.6\% (Joura et al., 2014; Merck } \\
\text { Sharp \& Dohme Corp., 2015) }\end{array}$ \\
CIN2/3 (proxy for & (Lehtinen et al., 2012) & & 62.8\% (Merck Sharp \& Dohme \\
CIN2/CIN2+) & (Lehtinen et al., 2012) & $42.7 \%$ (Munoz et al., 2010) & Corp., 2015) \\
CIN1 (proxy for CIN1/CIN1+ & $50.3 \%$ & $29.7 \%$ (Munoz et al., 2010) & $47.1 \%$ (Merck Sharp \& Dohme \\
or "any grade") & (Lehtinen et al., 2012) & & Corp., 2015) \\
GW & $0.0 \%$ (GSK Taiwan, 2016) & $82.8 \%$ (Munoz et al., 2010) & $94.6 \%$ (Giuliano et al., 2014) \\
\hline
\end{tabular}

4vHPVv, human papillomavirus 6/11/16/18 vaccine; 9vHPVv, human papillomavirus 6/11/16/18/31/33/45/52/58 vaccine; AS04-HPV-16/18v, AS04-adjuvanted HPV-16/18 vaccine; CC, cervical cancer; CIN1/2/3 (+), cervical intraepithelial neoplasia grade 1/2/3 (or higher); GW, genital warts 
Table 2. Age-Specific CC Incidence/Mortality and GW Incidence Per 100,000 Women in Taiwan

\begin{tabular}{|c|c|c|c|}
\hline Age-groups (years of age) & $\begin{array}{c}\text { CC incidence }(/ 100,000 \\
\text { women) (Health Promotion } \\
\text { Administration - Ministry of } \\
\text { Health and Welfare - Taiwan) }\end{array}$ & $\begin{array}{c}\text { CC mortality (/100,000 women) } \\
\text { (Health Promotion Administration } \\
\text { - Ministry of Health and } \\
\text { Welfare - Taiwan) }\end{array}$ & $\begin{array}{c}\text { GW incidence (/100,000 } \\
\text { women) (Ministry of } \\
\text { Health and } \\
\text { Welfare - Taiwan, 2013) }\end{array}$ \\
\hline$<15$ & NA & NA & 2.66 \\
\hline $15-19$ & 0.17 & 0.00 & 39.85 \\
\hline $20-24$ & 0.65 & 0.00 & 98.30 \\
\hline $25-29$ & 2.45 & 0.51 & 75.92 \\
\hline $30-34$ & 5.18 & 0.41 & 53.96 \\
\hline $35-39$ & 8.78 & 0.80 & 33.80 \\
\hline $40-44$ & 12.23 & 2.09 & 24.47 \\
\hline $45-49$ & 15.20 & 4.31 & 15.84 \\
\hline $50-54$ & 22.24 & 6.78 & 14.73 \\
\hline $55-59$ & 22.90 & 6.87 & 10.91 \\
\hline $60-64$ & 23.60 & 9.25 & 11.24 \\
\hline $65-69$ & 22.99 & 9.95 & 10.13 \\
\hline $70-74$ & 27.23 & 17.35 & 9.60 \\
\hline $75-79$ & 33.19 & 21.24 & 5.09 \\
\hline $80-84$ & 38.38 & 37.89 & 5.87 \\
\hline$\geq 85$ & 38.81 & 47.17 & 12.79 \\
\hline
\end{tabular}

CC, cervical cancer; GW, genital warts; NA: not applicable

AS04-HPV-16/18v+screening was associated with 0.0365 additional QALYs and increased costs of NT\$ 5,770 per person. This resulted in an ICER of NT\$ 158,157 per QALY gained, which fell below the threshold of NT\$ 727,818 (Directorate General of Budget - Accounting and Statistics - Executive Yuan - R.O.C. (Taiwan), 2017), indicating that the 2D-AS04-HPV-16/18v+screening is cost-effective compared with the screening programme alone (Table 5).

When compared with 2D-4vHPVv+screening, 2D-AS04-HPV-16/18v+screening was associated with 0.0201 additional QALYs and NT\$ 293 saved, per person. 2D-AS04-HPV-16/18v+screening also resulted in 0.0049 additional QALYs, compared with 2D/3D-

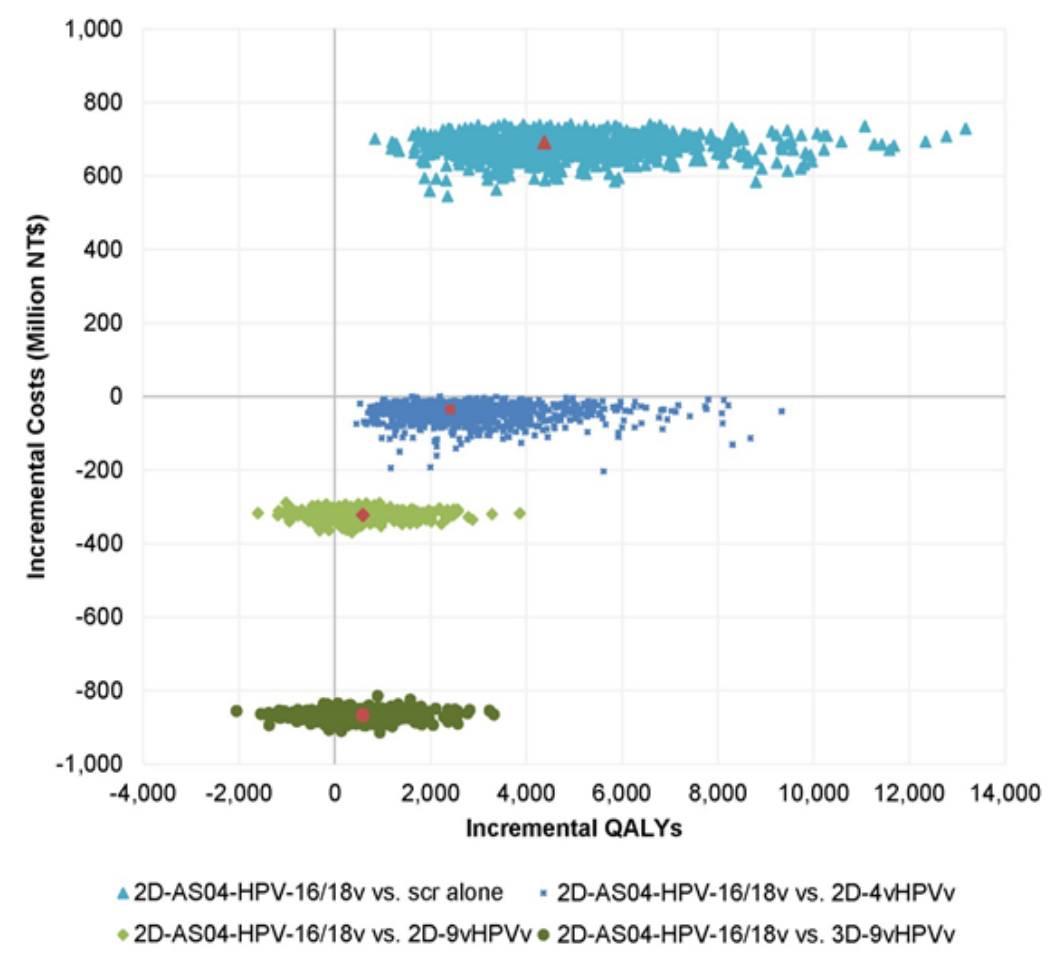

Figure 1. Probabilistic sensitivity analysis results. 2D, two-dose; 3D, three-dose; 4vHPVv, human papillomavirus 6/11/16/18 vaccine; 9vHPVv, human papillomavirus 6/11/16/18/31/33/45/52/58 vaccine; AS04-HPV-16/18v, AS04adjuvanted HPV-16/18 vaccine; HPV, human papillomavirus; NT\$, New Taiwan dollar; QALY, quality-adjusted life year; scr, screening 
Table 3. Cost-Per-Case Estimates Used in the Base-Case Analysis

\begin{tabular}{|c|c|c|}
\hline Parameter & Cost per case (NT\$) & References \\
\hline \multicolumn{3}{|l|}{ Treatment costs } \\
\hline Treatment cost of $\mathrm{CC}^{\mathrm{a}, \mathrm{b}}$ & 594,130 & $\begin{array}{l}\text { Taiwan Cancer Registry (Department of Statistics - Ministry of Health } \\
\text { and Welfare - Taiwan); NHID (Ministry of Health and Welfare - Tai- } \\
\text { wan, 2013) } \\
\text { Data examined: 01-Jan-2008 to 31-Dec-2015 }\end{array}$ \\
\hline $\begin{array}{l}\text { Treatment cost of } \\
\text { CIN } 2 / 3^{\text {b,c }}\end{array}$ & 11,245 & $\begin{array}{l}\text { Biopsy dataset (Pap Smear Task Force), NHID (Ministry of Health and } \\
\text { Welfare - Taiwan, 2013) } \\
\text { Data examined: 1-Jan-2011 to 31-Dec-2013 }\end{array}$ \\
\hline $\begin{array}{l}\text { Follow-up treatment cost } \\
\text { for CIN2/3,e }\end{array}$ & 2,266 & $\begin{array}{l}\text { National Health Insurance Administration - Ministry of Health and } \\
\text { Welfare - Taiwan (2016) }\end{array}$ \\
\hline Treatment cost of CIN $1^{\mathrm{b}, \mathrm{c}}$ & 2,962 & $\begin{array}{l}\text { Biopsy datasets (Pap Smear Task Force), Pap smear test datasets (Pap } \\
\text { Smear Task Force), NHID (Ministry of Health and Welfare - Taiwan, } \\
\text { 2013) } \\
\text { Data examined: 1-Jan-2011 to 31-Dec-2013 }\end{array}$ \\
\hline $\begin{array}{l}\text { Follow-up treatment cost } \\
\text { for CIN1 } 1^{\text {d,e }}\end{array}$ & 1,511 & $\begin{array}{l}\text { National Health Insurance Administration - Ministry of Health and } \\
\text { Welfare - Taiwan (2016) }\end{array}$ \\
\hline Treatment cost of $\mathrm{GW}^{\mathrm{b}, \mathrm{c}}$ & 6,170 & $\begin{array}{l}\text { NHID (Ministry of Health and Welfare - Taiwan, 2013) } \\
\text { Data examined: 1-Jan-2011 to 31-Dec-2013 }\end{array}$ \\
\hline \multicolumn{3}{|l|}{ Cost of regular Pap screening } \\
\hline $\begin{array}{l}\text { Woman with negative Pap } \\
\text { smear }^{\mathrm{f}}\end{array}$ & 430 & $\begin{array}{l}\text { National Health Insurance Administration - Ministry of Health and } \\
\text { Welfare - Taiwan (2016) }\end{array}$ \\
\hline $\begin{array}{l}\text { Woman with positive Pap } \\
\text { smear }^{\mathrm{f}}\end{array}$ & 2,966 & $\begin{array}{l}\text { National Health Insurance Administration - Ministry of Health and } \\
\text { Welfare - Taiwan (2016) }\end{array}$ \\
\hline \multicolumn{3}{|l|}{ Vaccination costs (per dose) $)^{g}$} \\
\hline AS04-HPV-16/18v & 3,600 & Centers for Disease Control - Taiwan (2016) \\
\hline 4vHPVv & 3,600 & Centers for Disease Control - Taiwan (2016) \\
\hline 9vHPVv & 5,100 & Centers for Disease Control - Taiwan (2016) \\
\hline \multicolumn{3}{|c|}{ 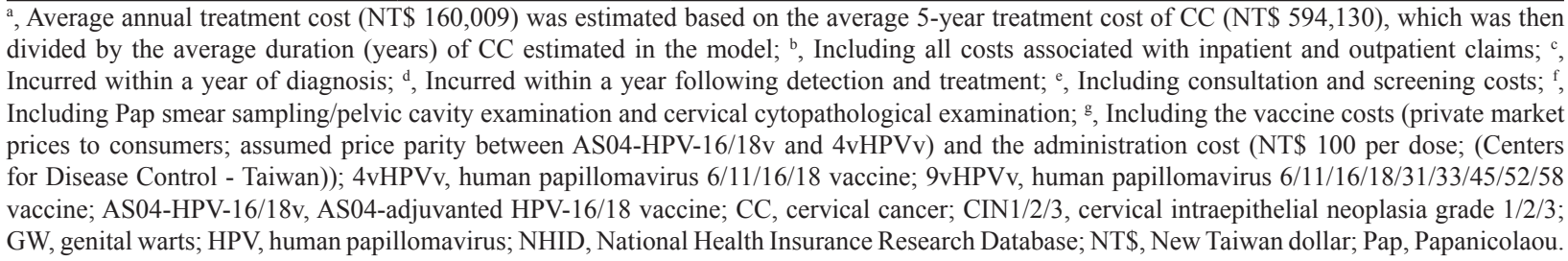 } \\
\hline
\end{tabular}

$9 \mathrm{vHPVv}+$ screening and cost savings of NT\$2,687 and NT\$ 7,226 per person, compared with the 2D and $3 \mathrm{D}-9 \mathrm{vHPVv}+$ screening, respectively. Therefore, 2D- AS04-HPV-16/18v+screening dominated (i.e. incurred greater QALYs at lower costs compared with) 2D-4vHPVv+screening and 2D/3D-9vHPVv+screening (Table 5).

\section{Scenario and sensitivity analysis}

Scenario analysis

When considering a limited duration of immunity, a higher GW incidence or vaccine tender prices, the 2D-AS04-HPV-16/18v+screening still remained costeffective compared with screening only, with NT\$ 168,163 , NT\$ 158,161 and NT\$ 70,335 per QALY gained, respectively.

All the scenarios considered led to the same conclusions as the base-case analysis of 2D-AS04-HPV$16 / 18 v+$ screening dominating all the other vaccines added to screening. Compared with 2D-4vHPVv+screening, 2D-9vHPVv+screening and 3D-9vHPVv+screening, 2D -AS04-HPV-16/18v+screening was still associated with

Table 4. QALY Loss Used in the Base-Case Analysis

\begin{tabular}{lcc}
\hline Health state & QALY loss & References \\
\hline Cancer cured & 0.1035 & Goldie et al. (2004), Taiwan Cancer Registry Center (2014) \\
Cancer treated & 0.3830 & Marcellusi et al. (2015), Annemans et al. (2009) \\
CIN2/3 detected & 0.0230 & Marcellusi et al. (2015), Annemans et al. (2009) \\
CIN1 detected & 0.0766 & Marcellusi et al. (2015), Annemans et al. (2009) \\
GW & 0.0396 & Marcellusi et al. (2015), Annemans et al. (2009) \\
\hline
\end{tabular}

CIN1, cervical intraepithelial neoplasia grade 1; CIN2/3, cervical intraepithelial neoplasia grade 2 or 3; GW, genital warts; QALY, quality-adjusted life year 
Table 5. Incremental Number of Cases, Total Costs, LYs and QALYs Gained (Per Person) for 2D-AS04-HPV16/18v+Screening vs Screening Only, 2D-4vHPVv+Screening and 2D/3D-9vHPVv+Screening

\begin{tabular}{|c|c|c|c|c|}
\hline \multirow[t]{2}{*}{ Results } & \multicolumn{4}{|c|}{ 2D-AS04-HPV-16/18v+screening compared with } \\
\hline & Screening only & $2 \mathrm{D}-4 \mathrm{vHPVv}+\mathrm{scr}$ & 2D-9vHPVv+scr & $3 \mathrm{D}-9 \mathrm{vHPVv}+\mathrm{scr}$ \\
\hline \multicolumn{5}{|l|}{ Undiscounted number of cases } \\
\hline $\mathrm{CC}$ cases & -0.0035 & -0.0020 & -0.0006 & -0.0006 \\
\hline CC deaths & -0.0010 & -0.0006 & -0.0002 & -0.0002 \\
\hline Screening-detected CIN $2 / 3$ cases & -0.0046 & -0.0026 & -0.0008 & -0.0008 \\
\hline Screening-detected CIN1 cases & -0.0371 & -0.0098 & 0.0101 & 0.0101 \\
\hline GW cases & 0.0000 & 0.0124 & 0.0144 & 0.0144 \\
\hline \multicolumn{5}{|c|}{ Discounted costs (NT\$) and health outcomes } \\
\hline Total cost & 5,770 & -293 & $-2,687$ & $-7,226$ \\
\hline LY gained & 0.0287 & 0.0167 & 0.0048 & 0.0048 \\
\hline QALY gained & 0.0365 & 0.0201 & 0.0049 & 0.0049 \\
\hline ICER per QALY gained (NT\$) & 158,157 & $\begin{array}{c}\text { AS04-HPV-16/18v+scr } \\
\text { dominates 2D- } \\
4 \mathrm{vHPV}+\mathrm{scr}\end{array}$ & $\begin{array}{c}\text { AS04-HPV-16/18v+scr } \\
\text { dominates 2D- } \\
9 \mathrm{vHPV}+\mathrm{scr}\end{array}$ & $\begin{array}{c}\text { AS04-HPV- } \\
\text { 16/18v+scr dominates } \\
\text { 3D-9vHPVv+scr }\end{array}$ \\
\hline
\end{tabular}

both cost savings (NT\$ 157-293, NT\$1,263-2,685 and NT\$3,488-7,224 per person, respectively) and additional QALYs gained (0.0191-0.0201, 0.0039-0.0049 and 0.0039-0.0049 per person, respectively).

Detailed results of scenario analyses are provided in Supplementary Material 8.

\section{Univariate sensitivity analysis}

The results of the univariate sensitivity analysis demonstrated the robustness of the base-case analysis results (Supplementary Material 9). For 2D-AS04-HPV$16 / 18 \mathrm{v}+$ screening versus screening alone, the results are summarised separately by the impact on costs, QALYs and the ICERs. For the five variables with the biggest impact on costs, QALYs and the ICERs, estimates per person are presented in Figure S 1 in Supplementary Material 9.

For the analyses comparing 2D-AS04-HPV$16 / 18 v+$ screening with $2 \mathrm{D}-4 \mathrm{vHPVv}+$ screening and 2D/3D-9vHPVv+screening, only the incremental costs and QALYs are reported separately as the incremental costs or QALYs are often negative (Supplementary Material 9).

The 2D-AS04-HPV-16/18v+screening remained costeffective compared with screening alone and dominant against the 2D-4vHPVv+screening, despite variations in the model inputs. The 2D-AS04-HPV-16/18v+screening also remained the dominant choice compared with the 2D/3D-9vHPVv+screening under all variable changes except when the model simultaneously assumed the lower limit of 2D-AS04-HPV-16/18v effectiveness and upper limit of $9 \mathrm{vHPVv}$ effectiveness. In this case, 2D-AS04HPV-16/18v+screening generated cost savings but was also less effective (Supplementary Material 6).

\section{Probabilistic sensitivity analysis}

The results of the PSA are shown as the scatter-plot figure in a cost-effectiveness plane presented in Figure 1.
For the comparison between 2D-AS04-HPV$16 / 18 \mathrm{v}+$ screening and screening alone, $100 \%$ of the replicates fell in quadrant I, with $99.9 \%$ below the threshold, indicating that vaccination with 2D-AS04HPV-16/18v is a cost-effective option. For the comparison between 2D-AS04-HPV-16/18v+screening and 2D$4 \mathrm{vHPVv}+$ screening, $99.9 \%$ of the replicates fell in quadrant $\mathrm{IV}$, indicating that 2D-AS04-HPV-16/18v+screening mostly dominates $2 \mathrm{D}-4 \mathrm{vHPVv}+$ screening while the remaining $0.1 \%$ fell in quadrant I below the threshold.

Regarding the comparison between 2D-AS04-HPV$16 / 18 \mathrm{v}+$ screening and 2D/3D-9vHPVv+screening, $78.0 \%$ and $76.4 \%$ of the replicates fell in quadrant IV, while $22.0 \%$ and $23.6 \%$ of the replicates fell in quadrant III, respectively.

\section{Discussion}

This study examines the cost-effectiveness of vaccination with AS04-HPV-16/18v+screening compared with screening alone and against other available HPV vaccines in Taiwan incorporating the new 2-dose schedules of AS04-HPV-16/18v and 4vHPVv, as well as the recently available vaccine $9 \mathrm{vHPVv}(2 \mathrm{D}$ and $3 \mathrm{D})$. Moreover, unlike previous studies, the present study focuses on the overall vaccine effectiveness irrespective of HPV type.

For the comparison of 2D-AS04-HPV$16 / 18 \mathrm{v}+$ screening with screening alone, in the basecase, 2D-AS04-HPV-16/18v+screening results in 0.0365 QALYs gained at an additional cost of NT\$ 5,770 per person after discounting. This results in an ICER of NT\$ 158,157 per QALY gained, which is considered cost-effective in Taiwan as the ICER falls below the threshold of NT\$ 727,818 (Directorate General of Budget - Accounting and Statistics - Executive Yuan - R.O.C. (Taiwan), 2017). When compared with 2D- 
$4 \mathrm{vHPVv}+$ screening and 2D/3D-9vHPVv+screening, 2D-AS04-HPV-16/18v+screening generates more QALYs (0.0201, 0.0049 and 0.0049 , respectively) at lower costs (-NT\$293, -NT\$2,687 and -NT\$ 7,226 respectively) per person. The 2D-AS04-HPV-16/18v+screening therefore dominates $2 \mathrm{D}-4 \mathrm{vHPVv}+$ screening and 2D/3D$9 \mathrm{vHPV} v+$ screening. These cost savings and better health outcomes seem to be predominantly driven by the superior vaccine effectiveness against $\mathrm{CC}$ with 2D-AS04-HPV16/18v compared with the other HPV vaccines available in Taiwan.

The findings of the present study are consistent with those from previous cost-effectiveness studies conducted in Taiwan that reported HPV vaccination as a costeffective option compared with screening alone (Dasbach et al., 2008; Liu et al., 2010; Rogoza et al., 2008; Suárez et al., 2008). They are also consistent with those from a previous cost-effectiveness study conducted in Taiwan concluding that AS04-HPV-16/18v+screening is the dominant choice compared with $4 \mathrm{vHPV}+$ screening (Demarteau et al., 2012).

The present study also reports that 2D-AS04HPV-16/18v+screening averts a greater number of screening-detected CIN2/3 cases, $\mathrm{CC}$ cases and CC deaths compared with 2D-4vHPVv+screening and 2D/3D$9 \mathrm{vHPV} v+$ screening but prevents fewer screening-detected CIN1 cases compared with 2D/3D-9vHPVv+screening. This is likely attributable to the lower progression rates of patients to CIN2/3 or CC with 2D-AS04-HPV$16 / 18 \mathrm{v}+$ screening, leading to a larger HPV infection-free population in the 2D-AS04-HPV-16/18v+screening cohort thus susceptible to develop CIN1, compared with the other vaccines.

Although this analysis primarily considered the effectiveness of HPV vaccines against $\mathrm{CC}$, the prevention of GW cases was also examined. The base-case assumes that 2D-AS04-HPV-16/18v does not offer protection against GW, as indicated in its label in Taiwan (GSK Taiwan, 2016). Thus, the base-case analysis results in 2D-4vHPVv+screening and 2D/3D-9vHPVv+screening averting more GW cases, compared with 2D-AS04-HPV$16 / 18 v+$ screening. However, evidence from a clinical trial and real-world studies demonstrated moderate efficacy of 2D-AS04-HPV-16/18v in preventing GW (Canvin et al., 2016; Public Health England, 2012; Szarewski et al., 2013). In England, since the national vaccination programme with AS04-HPV-16/18v was implemented in 2008 , there has been a decrease of $30.6 \%$ in new diagnoses of GW among young women aged 15-19 years (Canvin et al., 2016) and 20.8\% among women above 19 years old (Public Health England, 2012). This observation was supported by a post-hoc analysis of the randomised controlled trial (Szarewski et al., 2013). As such, the current base-case analysis is likely to underestimate the value of AS04-HPV-16/18v by assuming no protection against GW.

The robustness of the model and base-case results is demonstrated by the scenario and sensitivity analyses. Even when immunity starts declining after 20 years post-vaccination, or higher GW incidence (South Korean data) or vaccine tender prices instead of private market prices are used in the model, the results remain similar to the base-case analysis. Also, note that at the time of manuscript development, Cho et al., (2017) published GW incidence data for Taiwan. The GW incidence estimates presented in Cho et al., (2017) are quite different from the one used in the current study, due to the differing definitions of GW cases. Nonetheless, using the higher GW incidence from Cho et al., (2017) does not affect the conclusions (data not shown). In the univariate sensitivity analysis, despite change in the incremental costs and health outcomes from the variability in discount rates and vaccination costs, the overall conclusions of the base-case analysis broadly remain the same. The only exception is when the lower limit of 2D-AS04-HPV-16/18v effectiveness and the upper limit of 9vHPVv effectiveness were simultaneously assumed. In this situation 2D-AS04HPV-16/18v+screening generated cost savings but was also less effective. The PSA also confirms the robustness of the results. In the majority of cases, 2D-AS04-HPV$16 / 18 \mathrm{v}+$ screening is a cost-effective option compared with no vaccination and a dominant option compared with 2D-4vHPVv+screening and 2D/3D-9vHPVv+screening.

As in any economic analysis of vaccines, our model has several limitations. Unlike a transmission dynamic model, our static cohort model could not account for the benefits of herd immunity (Demarteau et al., 2012; Suárez et al., 2008). Our study also only focuses on the impact of HPV vaccines against $\mathrm{CC}$, in accordance with the WHO's key priority for HPV vaccines (World Health Organization, 2017), and did not distinguish between squamous cell carcinoma and adenocarcinoma. Moreover, our model does not consider vaccine protection against other HPV-related cancers such as vulvar, vaginal, anal or oropharyngeal carcinoma, and laryngeal papillomatosis. Consequently, the potential health benefits of the vaccines may have been underestimated.

In Taiwan, adding 2D-AS04-HPV-16/18v to the existing $\mathrm{CC}$ screening programme is expected to be costeffective compared with screening alone with estimated ICER falling below the WTP threshold. Compared with incorporating $2 \mathrm{D}-4 \mathrm{vHPVv}$ or $2 \mathrm{D} / 3 \mathrm{D}-9 \mathrm{vHPV}, \mathrm{HPV}$ vaccination using the $2 \mathrm{D}-\mathrm{AS} 04-\mathrm{HPV}-16 / 18 \mathrm{v}$ results in cost savings and greater QALY gains. Therefore, in addition to the current screening programme, 2D-AS04HPV-16/18v is the dominant choice when compared with all other available HPV vaccines in Taiwan.

\section{Acknowledgements}

The authors are grateful to Dr Chun-Ju Chiang (Taiwan Cancer Registry Center, Taiwan) and Ting-Yao Wang (Taipei Medical University, Taiwan) for their contribution to the study as well as Mei-Ling Chin (GSK, Taiwan), Stephen Griffiths (GSK, Belgium) and Rebecca Crawford (GSK, Singapore) for their support during the publication development. The authors would also like to thank Business \& Decision Life Sciences platform for editorial assistance and manuscript coordination, on behalf of GSK. Nathalie Arts coordinated publication development and provided editorial support and Sarah Fico provided medical writing support. 


\section{Disclosures}

\section{Contributorship}

All authors participated in the design or implementation or analysis, the interpretation of the study and the development of this manuscript. All authors had full access to the data and gave final approval before submission.

\section{Trademark section}

Cervarix is a trade mark owned by or licensed to the GSK group of companies. Gardasil is a trade mark of Merck and Co. Inc.

\section{Funding}

GlaxoSmithKline Biologicals SA was the funding source and was involved in all stages of the study conduct and analysis (GSK study identifier: HO-16-17794). GlaxoSmithKline Biologicals SA also took in charge all costs associated with the development and the publishing of the present manuscript.

\section{Conflicts of interest}

GVK, PM and CL are employees of the GSK group of companies. JYH was an employee of the GSK group of companies at the time of the study. WFC, SLY, LWH, JHJ and CHT have nothing to disclose.

\section{Focus on the Patient}

What is the context?

-Human papillomavirus (HPV) is the necessary cause of cervical cancer (CC).

-Three HPV vaccines are available in Taiwan: i) AS04HPV-16/18v (Cervarix, GSK), ii) 4vHPVv (Gardasil, Merck), and iii) 9vHPVv (Gardasil 9v, Merck).

-It is well-known that vaccination of adolescent girls along with screening plays an important role in reducing the risk of CC.

-Since the introduction of 2-dose (2D) schedules of AS04-HPV-16/18v and 4vHPVv, and the newly approved 2D/3D 9vHPVv, no published data are available on the relative cost-effectiveness of these vaccines in the context of Taiwan.

\section{What is new?}

-This study provides an updated cost-effectiveness analysis including recent data and incorporating the new schedules and vaccine.

-Moreover, unlike previous studies (that assessed vaccine effectiveness by HPV type) the present study focused on the overall vaccine effectiveness irrespective of HPV type.

What is the impact?

-These data suggest that adding 2D-AS04-HPV-16/18v to the existing screening programme is cost-effective compared with screening alone.

-2D-AS04-HPV-16/18v is the dominant option (better health outcomes at lower costs) compared with all other available HPV vaccines in Taiwan.

\section{References}

Annemans L, Remy V, Oyee J, Largeron N (2009).
Cost-effectiveness evaluation of a quadrivalent human papillomavirus vaccine in Belgium. Pharmacoeconomics, 27, 231-45.

Aregay M, Shkedy Z, Molenberghs G, David MP, Tibaldi F (2013). Model-based estimates of long-term persistence of induced HPV antibodies: a flexible subject-specific approach. J Biopharm Stat, 23, 1228-48.

Bertram MY, Lauer JA, De Joncheere K, et al (2016). Costeffectiveness thresholds: pros and cons. Bull World Health Organ, 94, 925-30.

Bureau of Health Promotion (2007). Pap Smear Screening Registry System Annual Report 2006. Report no. 19924695. Department of Health, Executive Yuan: Republic of China (Taiwan).

Cameron RL, Kavanagh K, Cameron Watt D, et al (2017). The impact of bivalent HPV vaccine on cervical intraepithelial neoplasia by deprivation in Scotland: reducing the gap. $J$ Epidemiol Community Health, 71, 954-60.

Canvin M, Sinka K, Hughes G, Mesher D (2016). Decline in genital warts diagnoses among young women and young men since the introduction of the bivalent HPV (16/18) vaccination programme in England: an ecological analysis. Sex Transm Infect, 93, 125-8.

Castellsague X (2008). Natural history and epidemiology of HPV infection and cervical cancer. Gynecol Oncol, 110, 4-7.

Center for Drug Evaluation (2014). Health Technology Assessment Methodology Guidelines. http://www.ispor.org/ PEguidelines/source/HTA_guidelines_Taiwan.pdf(accessed April 24, 2018).

Centers for Disease Control - Taiwan 106 (Centers for Disease Control Taiwan - From 2017, for every vaccine dose in the routine vaccination for children at and under 1 year of age, a vaccination fee of TWD 100 is subsidized.). http://www. cdc.gov.tw/info.aspx?treeid=45da8e73a81d495d\&nowtreei $\mathrm{d}=1$ bd193ed6dabaee6\&tid=8C78B8D0EF818F9B (accessed May 08, 2017).

Chao A, Hsu KH, Lai CH, et al (2008). Cervical cancer screening program integrating Pap smear and HPV DNA testing: A population-based study. Int J Cancer, 122, 2835-41.

Chen HC, You SL, Hsieh CY, et al (2011). Prevalence of genotype-specific human papillomavirus infection and cervical neoplasia in Taiwan: A community-based survey of 10,602 women. Int J Cancer, 128, 1192-203.

Cho CY, Lo YC, Hung MC, et al (2017). Risk of cancer in patients with genital warts: A nationwide, population-based cohort study in Taiwan. PLoS One, 12, e0183183.

Dasbach EJ, Insinga RP, Yang YC, et al (2008). The costeffectiveness of a quadrivalent human papillomavirus vaccine in Taiwan. Asian Pac J Cancer Prev, 9, 459-66.

David MP, Van HK, Hardt K, et al (2009). Long-term persistence of anti-HPV-16 and -18 antibodies induced by vaccination with the AS04-adjuvanted cervical cancer vaccine: modeling of sustained antibody responses. Gynecol Oncol, 115, 1-6.

Demarteau N, Tang CH, Chen HC, Chen CJ, Van Kriekinge G (2012). Cost-effectiveness analysis of the bivalent compared with the quadrivalent human papillomavirus vaccines in Taiwan. Value Health, 15, 622-31.

Department of Household Registration - M.O.I. (2016). Statistics. http://www.ris.gov.tw/en/web/ris3-english/endof-year (accessed April 24, 2018).

Department of Statistics - Ministry of Health and Welfare Taiwan Taiwan Cancer Registry Dataset.

Directorate General of Budget - Accounting and Statistics Executive Yuan - R.O.C. (Taiwan) (2017). Global reach - Achieving quality. https://eng.dgbas.gov.tw/mp.asp?mp=2 (accessed March 2, 2017).

Ferris D, Samakoses R, Block SL, et al (2014). Long-term study 
of a quadrivalent human papillomavirus vaccine. Pediatrics, 134, e657-65.

Gertig DM, Brotherton JM, Budd AC, et al (2013). Impact of a population-based HPV vaccination program on cervical abnormalities: a data linkage study. BMC Med, 11, 227.

Giuliano A, Joura E, Iversen O, et al (2014). Efficacy of a novel 9-valent HPV L1 vaccine against disease irrespective of HPV type. In: Proceedings of the 29th International Papillomavirus Conference and Clinical and Public Health Workshops; Aug 20-25, 2014; Seattle, Washington, USA. Accession no: PH.PD04.05, p 252.

Goldie SJ, Kohli M, Grima D, et al (2004). Projected clinical benefits and cost-effectiveness of a human papillomavirus 16/18 vaccine. J Natl Cancer Inst, 96, 604-15.

Government e-Procurement System for Health Promotion Administration, Ministry of Health and Welfare: 2016 working plan of Human Papillomavirus vaccines procurement and vaccination; award announcement (not awarded)[Updated as of September 10, 2016]. http://web. pcc.gov.tw/tps/main $/ \mathrm{pms} / \mathrm{tps} / \mathrm{atm} / \mathrm{atmNonAwardAction.do}$ ?searchMode $=$ common\&method $=$ nonAwardContentForP ublic\&pkAtmMain=51975053 (accessed August 11, 2017).

Government e-Procurement System for Public Health Bureau, Chiayi City: 2017 working plan of Chiayi City for Human Papillomavirus vaccines procurement and vaccination; award announcement [Updated as of April 28, 2017] http:// web.pcc.gov.tw/tps/main/pms/tps/atm/atmAwardAction.do? newEdit $=$ false\&searchMode $=$ common\&method=inquiryFo rPublic\&pkAtmMain=52125202\&tenderCaseNo=A106005 (accessed August 11, 2017).

GSK Taiwan (2016). Cervarix ${ }^{\circledR}$ Pescribing Information (Taiwan). https://www.fda.gov.tw/mlms/H0001D. aspx Type $=$ Lic\&LicId $=10000856$ (accessed January 30, 2018).

Health Promotion Administration - Ministry of Health and Welfare - Taiwan. Cancer Registry Interactive System. https://cris.hpa.gov.tw (accessed Januray 2, 2017).

Health Promotion Administration - Ministry of Health and Welfare - Taiwan (2014). Cervical Cancer Screening Registry System Annual Report. http://tcr.cph.ntu.edu.tw/ main.php?Page=A5 (accessed June 1, 2017).

International Society for Pharmacoeconomics and Outcome Research (2006). Guidelines of Methodological Standards for Pharmacoeconomic Evaluations in Taiwan. https://www. ispor.org/PEguidelines/source/2006_PEG_EN_2009.pdf (accessed January 15, 2018).

Joura EA, Ault KA, Bosch FX, et al (2014). Attribution of 12 high-risk human papillomavirus genotypes to infection and cervical disease. Cancer Epidemiol Biomarkers Prev, 23, 1997-2008.

Joura EA, Giuliano AR, Iversen OE, et al (2015). A 9-valent HPV vaccine against infection and intraepithelial neoplasia in women. $N$ Engl J Med, 372, 711-23.

Kavanagh K, Pollock KG, Cuschieri K, et al (2017). Changes in the prevalence of human papillomavirus following a national bivalent human papillomavirus vaccination programme in Scotland: a 7-year cross-sectional study. Lancet Infect Dis, 17, 1293-302.

Köse FM, Naki MM (2014). Cervical premalignant lesions and their management. J Turk Ger Gynecol Assoc, 15, 109-21.

Lee CC, Chen TS, Wu TZ, Huang LM (2012). A human papillomavirus public vaccination program in Taiwan: the Kinmen County experience. J Formos Med Assoc, 111, 682-5.

Lehtinen M, Paavonen J, Wheeler CM, et al (2012). Overall efficacy of HPV-16/18 AS04-adjuvanted vaccine against grade 3 or greater cervical intraepithelial neoplasia: 4-year end-of-study analysis of the randomised, double-blind PATRICIA trial. Lancet Oncol, 13, 89-99.

Liu PH, Hu FC, Lee PI, et al (2010). Cost-effectiveness of human papillomavirus vaccination for prevention of cervical cancer in Taiwan. BMC Health Serv Res, 10, 11.

Marcellusi A, Capone A, Favato G, et al (2015). Health utilities lost and risk factors associated with HPV-induced diseases in men and women: the HPV Italian collaborative study group. Clin Ther, 37, 156-67.e4.

Merck Sharp and Dohme Corp. (2015). Clinical Study Report P001. http://198.61.244.207/Upload/83_Applied\%20 Redaction\%20V503\%20P001\%20CSR\%20Synopsis.pdf (accessed 15/01/2018).

Ministry of Health and Welfare - Taiwan (2013). National Health Insurance Research Database. https://www.moi.gov.tw/stat/ node.aspx?cate_sn $=\&$ belong_sn $=6189 \& s n=6190($ accessed June 1, 2017).

Ministry of the Interior - Department of Statistics. Life table in Taiwan. http://www.moi.gov.tw/stat/life.aspx (accessed February 20, 2017).

Munoz N, Kjaer SK, Sigurdsson K, et al (2010). Impact of human papillomavirus (HPV)-6/11/16/18 vaccine on all HPV-associated genital diseases in young women. $J$ Natl Cancer Inst, 102, 325-39.

Muñoz N, Bosch FX, de Sanjosé S, et al (2003). Epidemiologic classification of human papillomavirus types associated with cervical cancer. $N$ Engl J Med, 348, 518-27.

National Evidence-based Healthcare Collaborating Agency (NECA) Study (2012). Economic Evaluation of Human Papillomavirus Vaccines. https://www.neca.re.kr/lay1/ program/S1T11C145/report/view.do?seq=88 (accessed June 01, 2017).

National Health Insurance Administration - Ministry of Health and Welfare - Taiwan (2016). National Health Insurance (NHI) fee schedule. http://www.nhi.gov.tw/English/ webdata/webdata.aspx?menu=11\&menu_id=591\&WD_ ID=591\&webdata_id=3633 (accessed April 10, 2017).

National Statistics - Republic of China (Taiwan) (2018). Consumer Price Indices. https://eng.stat.gov.tw/ct.asp?xIte $\mathrm{m}=12092 \& \mathrm{ctNode}=1558 \& \mathrm{mp}=5$ (accessed June 13, 2018).

Naud PS, Roteli-Martins CM, De Carvalho NS, et al (2014). Sustained efficacy, immunogenicity, and safety of the HPV-16/18 AS04-adjuvanted vaccine: final analysis of a long-term follow-up study up to 9.4 years post-vaccination. Hum Vaccin Immunother, 10, 2147-62.

Paavonen J, Naud P, Salmeron J, et al (2009). Efficacy of human papillomavirus (HPV)-16/18 AS04-adjuvanted vaccine against cervical infection and precancer caused by oncogenic HPV types (PATRICIA): final analysis of a double-blind, randomised study in young women. Lancet, 374, 301-14.

Pap Smear Task Force Biopsy Dataset.

Pap Smear Task Force Pap Smear Screening Dataset.

Powell SE, Hariri S, Steinau M, et al (2012). Impact of human papillomavirus (HPV) vaccination on HPV 16/18-related prevalence in precancerous cervical lesions. Vaccine, $\mathbf{3 1}$, 109-13.

Public Health England (2012). Health Protection Report - Sexually transmitted infections in England, 2011. http://webarchive. nationalarchives.gov.uk/20140714091348/http://www.hpa. org.uk/hpr/archives/2012/hpr2212.pdf (accessed January 29, 2018).

Richardson H, Kelsall G, Tellier P, et al (2003). The natural history of type-specific human papillomavirus infections in female university students. Cancer Epidemiol Biomarkers Prev, 12, 485-90.

Rogoza RM, Ferko N, Bentley J, et al (2008). Optimization of primary and secondary cervical cancer prevention strategies 
in an era of cervical cancer vaccination: a multi-regional health economic analysis. Vaccine, 26, 46-58.

Sanders GD, Taira AV (2003). Cost-effectiveness of a potential vaccine for human papillomavirus. Emerg Infect Dis, 9, $37-48$.

Shen S-C, Hung Y-C, Kung P-T, et al (2016). Factors involved in the delay of treatment initiation for cervical cancer patients: A nationwide population-based study. Medicine, 95, e4568.

Suárez E, Smith JS, Bosch FX, et al (2008). Cost-effectiveness of vaccination against cervical cancer: a multi-regional analysis assessing the impact of vaccine characteristics and alternative vaccination scenarios. Vaccine, 26, 29-45.

Szarewski A, Skinner SR, Garland SM, et al (2013). Efficacy of the HPV-16/18 AS04-adjuvanted vaccine against low-risk HPV types (PATRICIA randomized trial): an unexpected observation. J Infect Dis, 208, 1391-6.

Tabrizi SN, Brotherton JM, Stevens MP, et al (2014). HPV genotype prevalence in Australian women undergoing routine cervical screening by cytology status prior to implementation of an HPV vaccination program. J Clin Virol, 60, 250-6.

Taiwan Cancer Registry Center (2014). Taiwan Cancer Registry Annual Report. http://tcr.cph.ntu.edu.tw/main.php?Page=A5 (accessed August 1, 2017).

Toft L, Tolstrup M, Muller M, et al (2014). Comparison of the immunogenicity of Cervarix(R) and Gardasil(R) human papillomavirus vaccines for oncogenic non-vaccine serotypes HPV-31, HPV-33, and HPV-45 in HIV-infected adults. Hum Vaccin Immunother, 10, 1147-54.

Van de Velde N, Brisson M, Boily MC (2007). Modeling human papillomavirus vaccine effectiveness: quantifying the impact of parameter uncertainty. Am J Epidemiol, 165, 762-75.

Walboomers JMM, Jacobs MV, Manos MM, et al (1999). Human papillomavirus is a necessary cause of invasive cervical cancer worldwide. J Pathol, 189, 12-9.

Wang PD, Lin RS (1996). Epidemiology of cervical cancer in Taiwan. Gynecol Oncol, 62, 344-52.

Woodhall SC, Jit M, Soldan K, et al (2011). The impact of genital warts: loss of quality of life and cost of treatment in eight sexual health clinics in the UK. Sex Transm Infect, 87, 458-63.

World Health Organization (2017). Human papillomavirus vaccines: WHO position paper, May 2017. Wkly Epidemiol Rec, 92, 241-68.

\section{c) (1) (8)}

This work is licensed under a Creative Commons AttributionNon Commercial 4.0 International License. 